Report no. $10 / 14$

\title{
A New Approximation of the Schur Complement in Preconditioners for PDE Constrained Optimization
}

\author{
John W. Pearson and Andrew J. Wathen
}

\begin{abstract}
Saddle point systems arise widely in optimization problems with constraints. The utility of Schur complement approximation is now broadly appreciated in the context of solving such saddle point systems by iteration. In this short manuscript, we present a new Schur complement approximation for PDE constrained optimization, an important class of these problems. Block diagonal and block triangular preconditioners have previously been designed to be used to solve such problems along with MINRES and non-standard Conjugate Gradients respectively; with appropriate approximation blocks these can be optimal in the sense that the time required for solution scales linearly with the problem size, however small the mesh size we use. In this paper, we extend this work to designing such preconditioners for which this optimality property holds independently of both the mesh size and of the Tikhonov regularization parameter $\beta$ that is used. This also leads to an effective symmetric indefinite preconditioner that exhibits mesh and $\beta$-independence. We motivate the choice of these preconditioners based on observations about approximating the Schur complement obtained from the matrix system, derive eigenvalue bounds which verify the effectiveness of the approximation, and present numerical results which show that these new preconditioners work well in practice.
\end{abstract}

Key words and phrases: PDE constrained optimization; Poisson control; preconditioning; Schur complement.

Oxford University Mathematical Institute

Numerical Analysis Group

24-29 St Giles'

Oxford, England OX1 3LB

E-mail: john.pearson@maths.ox.ac.uk

November, 2010 


\section{Introduction}

As with many other problems involving optimization with constraints, PDE constrained optimization problems lead to linear systems of equations with saddle point structure [1]. In the case of PDE constrained optimization, these matrices are typically of very large dimension, and iterative methods are usually employed for their solution (see $[2$, $8,10]$ ). It is now well understood $[1,6]$ that a key component in most preconditioning strategies for such saddle point problems is a good Schur complement approximation.

In this manuscript we consider tracking type PDE constrained optimization problems of the form

$$
\begin{aligned}
& \min _{y, u} \frac{1}{2}\|y-\hat{y}\|_{L_{2}(\Omega)}^{2}+\frac{\beta}{2}\|u\|_{L_{2}(\Omega)}^{2} \\
& \text { s.t. } \quad \mathcal{L} y=u \text { in } \Omega \text {, } \\
& y=g \quad \text { on } \partial \Omega \text {, }
\end{aligned}
$$

where $y$ is the state, $\hat{y}$ is a desired state, $u$ is the control, $\beta>0$ is a regularization (or Tikhonov) parameter, and $\Omega$ is the domain with boundary $\partial \Omega$. We assume that $\mathcal{L}$ denotes a positive definite elliptic operator, which for simplicity we will take to be the negative of the Laplacian in this manuscript. The results presented here apply more generally. Here the control is distributed throughout the domain, but control on subdomains or boundaries can also be of interest; also $\hat{y}$ may be defined only on a subdomain (see [15]). Our primary interest is in the construction of appropriate block preconditioners, and in particular in the definition of Schur complement approximations, for use in block diagonal or block triangular preconditioners. In [8], Rees, Dollar and Wathen describe such Schur complement approximations which are effective for moderately large values of $\beta$, but the performance of which deteriorates as $\beta \rightarrow 0$. In their construction of a block preconditioner for use with a non-standard inner product, Schöberl and Zulehner [10] pay particular attention to achieving robust behaviour with respect to small values of $\beta$.

Here we describe a new Schur complement approximation for PDE constrained optimization problems which can be employed in these (and other) approaches, and which yields convergence of the appropriate iterative method in a number of steps which is independent of the value of the regularization parameter $\beta$. We prove the relevant eigenvalue bounds which guarantee this property.

For the numerical solution of (1.1), we introduce a Lagrange multiplier (or adjoint variable) $p$, and assume that equal order finite element approximations are used for $y$, $u$ and $p$. Then, whichever of the discretize-then-optimize or optimize-then-discretize approach is used, the first order stationarity conditions (or KKT conditions) for the tracking problem (1.1) become the saddle point problem $[8,15]$

$$
\left[\begin{array}{ccc}
M & 0 & K \\
0 & \beta M & -M \\
K & -M & 0
\end{array}\right]\left[\begin{array}{l}
\mathbf{y} \\
\mathbf{u} \\
\mathbf{p}
\end{array}\right]=\left[\begin{array}{l}
\mathbf{b} \\
\mathbf{0} \\
\mathbf{d}
\end{array}\right]
$$


In (1.2), $K$ denotes the matrix representing the discrete differential operator, $M$ denotes a Grammian matrix (or mass matrix) of the relevant finite element basis, and $\mathbf{b}$ and $\mathbf{d}$ are vectors containing terms arising from $\hat{y}$ and the boundary terms of $y$ respectively. The vectors $\mathbf{y}, \mathbf{u}$ and $\mathbf{p}$ are the discretized versions of $y, u$ and $p$ respectively, precisely vectors of coefficients for the respective expansions in terms of the finite element basis functions used. A derivation for this system is given in [8]. The first, second and third block rows of this matrix system are sometimes referred to as the discretized versions of the adjoint equation, gradient equation and state equation respectively [15].

This paper is structured as follows. In Section 2, we review three preconditioners that have been proposed for solving the matrix system arising from the Poisson control problem (which is (1.2), with $K$ equal to a finite element stiffness matrix). These are block diagonal, block triangular and symmetric indefinite preconditioners respectively. In Section 3, we propose a new approximation to the Schur complement which, along with a good approximation to the mass matrix $M$, can be incorporated into any of the three preconditioners of Section 2. We demonstrate analytically why our approximation to the Schur complement is effective. In Section 4, we present numerical results to show how well our approximation works within the three preconditioners in practice, and in Section 5, we make some concluding remarks.

\section{Preconditioners for Poisson Control}

In this Section, we describe research that has been undertaken to solve the Poisson control problem: that is the problem (1.1) with $\mathcal{L}=-\nabla^{2}$. In Section 2.1, we describe in general terms why certain preconditioners are effective for this problem, based on its saddle point structure. We then detail three preconditioners that have been proposed for this problem; block triangular preconditioners (in Section 2.2), block triangular preconditioners (in Section 2.3) and symmetric indefinite preconditioners (in Section 2.4). We note the Schur complement approximations used in these preconditioners. This work will later be incorporated when testing the Schur complement approximation that we propose in Section 3.

\subsection{Background}

The problem we will solve is of saddle point form, that is

$$
\underbrace{\left[\begin{array}{cc}
A & B^{T} \\
B & 0
\end{array}\right]}_{\mathcal{A}}\left[\begin{array}{l}
\mathbf{x}_{1} \\
\mathbf{x}_{2}
\end{array}\right]=\left[\begin{array}{l}
\mathbf{b}_{1} \\
\mathbf{b}_{2}
\end{array}\right] .
$$

A review of solution methods for systems of the form (2.1) is given in [1].

It can be easily shown, as in [6], that if we consider the following 'ideal' preconditioners $\mathcal{P}_{1}$ and $\mathcal{P}_{2}$ for $\mathcal{A}$ :

$$
\mathcal{P}_{1}=\left[\begin{array}{cc}
A & 0 \\
0 & B A^{-1} B^{T}
\end{array}\right], \quad \mathcal{P}_{2}=\left[\begin{array}{cc}
A & 0 \\
B & -B A^{-1} B^{T}
\end{array}\right],
$$


then the spectra of $\mathcal{P}_{1}^{-1} \mathcal{A}$ and $\mathcal{P}_{2}^{-1} \mathcal{A}$ are

$$
\lambda\left(\mathcal{P}_{1}^{-1} \mathcal{A}\right)=\left\{1, \frac{1}{2}(1 \pm \sqrt{5})\right\}, \quad \lambda\left(\mathcal{P}_{2}^{-1} \mathcal{A}\right)=\{1\}
$$

provided that the preconditioned systems are nonsingular. In this case, $\mathcal{P}_{1}^{-1} \mathcal{A}$ is diagonalizable, but $\mathcal{P}_{2}^{-1} \mathcal{A}$ is not. The quantity $B A^{-1} B^{T}$, which appears in both of the preconditioners above, is the Schur complement, which we will denote by $S$ for the remainder of this manuscript.

The fact that we obtain these eigenvalue properties if we precondition a matrix $\mathcal{A}$ with $\mathcal{P}_{1}$ or $\mathcal{P}_{2}$ implies that an appropriate Krylov subspace method will converge in $3 / 2$ iterations respectively [6]. Of course implementing such a method in practice would involve exactly forming $S$ and finding the exact solution of linear systems with both $A$ and $S$. This would in general be wasteful, or indeed infeasible, since $S$ would generally be dense even when $A$ and $B$ are sparse. However, we can instead proceed by constructing approximations $\hat{A}$ and $\hat{S}$ to $A$ and $S$ respectively, and apply the preconditioners

$$
\hat{\mathcal{P}}_{1}=\left[\begin{array}{cc}
\hat{A} & 0 \\
0 & \hat{S}
\end{array}\right], \quad \hat{\mathcal{P}}_{2}=\left[\begin{array}{cc}
\hat{A} & 0 \\
B & -\hat{S}
\end{array}\right]
$$

The formulations of the preconditioners $\hat{\mathcal{P}}_{1}$ and $\hat{\mathcal{P}}_{2}$ motivate our discussion in Sections 2.2 and 2.3 respectively.

A third option is to construct a preconditioner for the matrix system (2.1) of the form

$$
\hat{\mathcal{P}}_{3}=\left[\begin{array}{cc}
\hat{A} & B^{T} \\
B & B \hat{A}^{-1} B^{T}-\hat{S}
\end{array}\right]=\left[\begin{array}{cc}
I & 0 \\
B \hat{A}^{-1} & I
\end{array}\right]\left[\begin{array}{cc}
\hat{A} & B^{T} \\
0 & -\hat{S}
\end{array}\right]
$$

meaning that one application of the approximation of $\hat{S}$ and two of $\hat{A}$ are required to effect this preconditioner. We will discuss this preconditioner further in Section 2.4.

In Sections 2.2-2.4, we consider three 'optimal' preconditioners, one each of the form $\hat{\mathcal{P}}_{1}, \hat{\mathcal{P}}_{2}$ and $\hat{\mathcal{P}}_{3}$. The word 'optimal' is used to mean that an appropriate Krylov subspace method with the preconditioner has linear complexity in matrix size, or alternatively that the number of iterations required for convergence of the solver is bounded independently of the mesh, with linear work required for each iteration. The solver detailed in Section 2.4 was designed to yield $\beta$-independent convergence as well as mesh-independent convergence.

\subsection{Symmetric positive definite (block diagonal) precondition- ers}

We apply the theory of Section 2.1 to the discrete Poisson control problem (1.2), where $A=\left[\begin{array}{cc}M & 0 \\ 0 & \beta M\end{array}\right]$ and $B=\left[\begin{array}{cc}K & -M\end{array}\right]$ in terms of the notation of (2.1). We may apply 
the block diagonal preconditioner

$$
\hat{\mathcal{P}}_{1}=\left[\begin{array}{ccc}
\hat{M} & 0 & 0 \\
0 & \beta \hat{M} & 0 \\
0 & 0 & \hat{S}
\end{array}\right]
$$

to the matrix system in (1.2), where $\hat{M}$ and $\hat{S}$ are approximations to the mass matrix $M$ and the Schur complement $S$ respectively. These approximations must be symmetric and positive definite, thus $\hat{\mathcal{P}}_{1}$ is symmetric and positive definite, and so it is possible to build this into a Krylov subspace algorithm for symmetric matrices such as MINRES [7]. In this way we exploit the advantages of symmetric Krylov subspace solvers, as detailed in Chapter 6 of [5].

Rees, Dollar and Wathen [8] introduced the block diagonal preconditioner (2.3) for PDE constrained optimization; they took the mass matrix approximation $\hat{M}$ to be a fixed number of steps of Chebyshev semi-iteration [17], exploiting the fact that $\operatorname{diag}(M)^{-1} M$ is very well conditioned for all commonly used finite element basis functions [16]. This highly effective method for preconditioning mass matrices is discussed further in [17]. The Schur complement approximation

$$
S=K M^{-1} K+\frac{1}{\beta} M \approx K M^{-1} K=: \hat{S}_{1}
$$

is motivated by the fact that the $\frac{1}{\beta} M$ is a higher order term in the step size $h$ than $K M^{-1} K$. (Theorem 3 gives upper and lower bounds for $\hat{S}_{1}^{-1} S$.) Multigrid cycles are employed to approximate $K$ each time, and a matrix multiplication to represent $M$, in each application of $\hat{S}_{1}$. This approximation leads to mesh-independent convergence, but convergence is not $\beta$-independent. The preconditioner we recommend in Section 3 enables us to overcome this issue, so that our method is insensitive to the value of $\beta$.

\subsection{Nonsymmetric positive definite (block triangular) precon- ditioners}

When employing the block triangular preconditioner $\hat{\mathcal{P}}_{2}$ in $(2.2)$, there is no simple equivalent symmetric formulation, since the matrix $\hat{\mathcal{P}}_{2}$ is not symmetric. This means that a method such as MINRES or conjugate gradients with a standard inner product cannot be used with this preconditioner. However, as outlined in $[4,11,18]$ and discussed in the context of Poisson control by Rees and Stoll in [9], $\hat{\mathcal{P}}_{2}^{-1} \mathcal{A}$ is self-adjoint and positive definite in the $\mathcal{H}$-inner product, defined as $\langle\mathbf{u}, \mathbf{v}\rangle_{\mathcal{H}}=\mathbf{u}^{T} \mathcal{H} \mathbf{v}$, where

$$
\mathcal{H}=\left[\begin{array}{cc}
A-\hat{A} & 0 \\
0 & \hat{S}
\end{array}\right] .
$$

One is therefore able to apply the Conjugate Gradient method with this non-standard inner product, a method known as the Bramble-Pasciak Conjugate Gradient method. 
Note that $A-\hat{A}$ must be positive definite for this to define an inner product; sometimes scaling is required to achieve this.

In [9], the application of this method to Poisson control is discussed. In this context, we may write the preconditioner as

$$
\hat{\mathcal{P}}_{2}=\left[\begin{array}{ccc}
\gamma \hat{M} & 0 & 0 \\
0 & \beta \gamma \hat{M} & 0 \\
K & -M & -\hat{S}
\end{array}\right]
$$

and the inner product as

$$
\mathcal{H}=\left[\begin{array}{ccc}
M-\gamma \hat{M} & 0 & 0 \\
0 & \beta(M-\gamma \hat{M}) & 0 \\
0 & 0 & \hat{S}
\end{array}\right],
$$

for a parameter $\gamma$ which ensures that $M-\gamma \hat{M}$ is positive definite.

As discussed in [9], it is effective to again take $\hat{M}$ as a fixed number of Chebyshev semi-iterations, and $\hat{S}$ as two multigrid approximations and a matrix multiplication. In [9] $\hat{S}$ was again taken to be $\hat{S}_{1}$ as in (2.4). Particular emphasis is given in [9] of the ease of choosing $\gamma$ to ensure that $M-\gamma \hat{M}$ is positive definite for the PDE contrained optimization problem.

\subsection{Symmetric indefinite preconditioners with block structure}

In [10], a preconditioner of the form $\hat{\mathcal{P}}_{3}$ is proposed for use with the Conjugate Gradient method in a non-standard inner product. Here, $\hat{A}$ and $\hat{S}$ are chosen such that the preconditioned system is positive definite with respect to the inner product $\overline{\mathcal{H}}$, where

$$
\langle\mathbf{u}, \mathbf{v}\rangle_{\overline{\mathcal{H}}}=\mathbf{u}^{T} \underbrace{\left[\begin{array}{cc}
\hat{A}-A & 0 \\
0 & B \hat{A}^{-1} B^{T}-\hat{S}
\end{array}\right]}_{\overline{\mathcal{H}}} \mathbf{v} .
$$

Applying this method to the Poisson control problem, Schöberl and Zulehner [10] took approximations

$$
\hat{A}=\frac{1}{\sigma} \hat{A}_{0}, \quad \hat{S}=\frac{1}{\tau} \hat{S}_{0}
$$

with

$$
\hat{A}_{0}=\left[\begin{array}{cc}
\hat{Y} & 0 \\
0 & \beta \hat{M}
\end{array}\right], \quad \hat{S}_{0}=\frac{\sigma}{\beta} \hat{Y}
$$

Here, $\hat{Y}$ denotes a multigrid solver applied to the matrix $\sqrt{\beta} K+M$, and $\hat{M}$ denotes application of the symmetric Gauss-Seidel method to the appropriate mass matrix. (Throughout the remainder of this manuscript, we will replace this mass matrix approximation 
with the Chebyshev semi-iteration method described above, as this is an effective and well-founded technique.) The formulation of (2.6) and (2.7) guarantees that

$$
\frac{1}{\sigma} \hat{A}_{0}>A, \quad \frac{1}{\tau} \hat{S}_{0}<B \hat{A}_{0}^{-1} B^{T},
$$

and hence that the preconditioned system is positive definite in the inner product $\overline{\mathcal{H}}$. The overall preconditioner for the Poisson control problem therefore looks as follows:

$$
\begin{aligned}
\hat{\mathcal{P}}_{3} & =\left[\begin{array}{cccc}
\frac{1}{\sigma} \hat{Y} & 0 & K \\
0 & \frac{\beta}{\sigma} \hat{M} & -M \\
K & -M & \sigma K \hat{Y}^{-1} K+\frac{\sigma}{\beta} M \hat{M}^{-1} M-\frac{\sigma}{\tau \beta} \hat{Y}
\end{array}\right] \\
& =\left[\begin{array}{ccc}
I & 0 & 0 \\
0 & I & 0 \\
\sigma K \hat{Y}^{-1} & -\frac{\sigma}{\beta} M \hat{M}^{-1} & I
\end{array}\right]\left[\begin{array}{ccc}
\frac{1}{\sigma} \hat{Y} & 0 & K \\
0 & \frac{\beta}{\sigma} \hat{M} & -M \\
0 & 0 & -\frac{\sigma}{\tau \beta} \hat{Y}
\end{array}\right] .
\end{aligned}
$$

In [10], it is recommended that $\sigma \approx 1$ and $\tau \approx \frac{4}{3}$, and these are the values we use in the computations of Section 4.

\section{An Improved Approximation of the Schur Com- plement}

All three methods detailed in Section 2 rely heavily on an accurate approximation of the Schur complement. When the block diagonal preconditioner for MINRES and block triangular preconditioner for Bramble-Pasciak detailed in Section 2 are applied, the iteration count becomes prohibitively large for small values of $\beta$. This is due to the neglected $\frac{1}{\beta} M$ term in the Schur complement approximation (2.4). A detailed computational analysis of the asymptotic behaviour of eigenvalue properties of the system (1.2) for small values of $\beta$ is given in $[13,14]$. In this Section, we introduce an alternative Schur complement approximation that we prove to give solvers robust to small values of $\beta$ for each of the approaches described in the previous Section.

Instead of (2.4), it is easily checked that we can write

$$
S=\left(K+\frac{1}{\sqrt{\beta}} M\right) M^{-1}\left(K+\frac{1}{\sqrt{\beta}} M\right)-\frac{2}{\sqrt{\beta}} K,
$$

which enables us to take the approximation

$$
S \approx \hat{S}_{2}=\left(K+\frac{1}{\sqrt{\beta}} M\right) M^{-1}\left(K+\frac{1}{\sqrt{\beta}} M\right)
$$

by dropping the $-\frac{2}{\sqrt{\beta}} K$ term. We highlight that the term discarded here is $\mathcal{O}\left(\beta^{-1 / 2}\right)$ rather than $\mathcal{O}\left(\beta^{-1}\right)$, as was the case in the Schur complement approximation $\hat{S}_{1}$. When 
we wish to use preconditioners involving the approximate Schur complement, the factorization (3.1) enables us to apply multigrid on two occasions to the matrix $K+\frac{1}{\sqrt{\beta}} M$ rather than the matrix $K$, together, as before, with a mass matrix multiplication. We note the similarity of this to applying the multigrid process $\hat{Y}$ to the matrix $\sqrt{\beta} K+M$ as discussed in Section 2.4, but note also that the multigrid cycles are applied at different points in the preconditioners.

We demonstrate theoretically why this approximation is more potent than the approximation $\hat{S}_{1}$ in (2.4). Note that Schöberl and Zulehner derive the different Schur complement approximation (2.6), (2.7) in [10]; we will demonstrate in Section 4 that our approximation (2.6), (3.1) also fits nicely into the preconditioning framework developed in [10]. Our analysis also applies to any other differential operator which satisfies these conditions.

In order to obtain convergence bounds or estimates, we wish to derive eigenvalue bounds for $\hat{S}_{1}^{-1} S$ and $\hat{S}_{2}^{-1} S$. To calculate the eigenvalues for the former, we first note Theorems 1 and 2, which are stated on pages 57-60 of [5].

Theorem 1 For the problem (1.2) for $\Omega \subset \mathbb{R}^{2}$, with a degree of approximation $\mathbf{P}_{m}$ or $\mathbf{Q}_{m}$ with $m \geq 1$, the following bound holds:

$$
c_{1} h^{2} \leq \frac{\mathbf{v}^{T} M \mathbf{v}}{\mathbf{v}^{T} \mathbf{v}} \leq C_{1} h^{2}, \quad \forall \mathbf{v} \in \mathbb{R}^{n},
$$

where the constants $c_{1}$ and $C_{1}$ are independent of the step size $h$ but dependent on $m$. For $\Omega \subset \mathbb{R}^{3}$, the equivalent result is

$$
c_{1} h^{3} \leq \frac{\mathbf{v}^{T} M \mathbf{v}}{\mathbf{v}^{T} \mathbf{v}} \leq C_{1} h^{3}, \quad \forall \mathbf{v} \in \mathbb{R}^{n}
$$

Theorem 2 For the problem (1.2) for $\Omega \subset \mathbb{R}^{2}$, with a degree of approximation $\mathbf{P}_{m}$ or $\mathbf{Q}_{m}$ with $m \geq 1$, the following bound holds:

$$
c_{2} h^{2} \leq \frac{\mathbf{v}^{T} K \mathbf{v}}{\mathbf{v}^{T} \mathbf{v}} \leq C_{2}, \quad \forall \mathbf{v} \in \mathbb{R}^{n},
$$

where the constants $c_{2}$ and $C_{2}$ are independent of the step size $h$ but dependent on $m$. For $\Omega \subset \mathbb{R}^{3}$, the equivalent result is

$$
c_{2} h^{3} \leq \frac{\mathbf{v}^{T} K \mathbf{v}}{\mathbf{v}^{T} \mathbf{v}} \leq C_{2} h, \quad \forall \mathbf{v} \in \mathbb{R}^{n}
$$

Theorems 1 and 2 give us that in $2 \mathrm{D}$ or $3 \mathrm{D}$, for any $\mathbf{v} \in \mathbb{R}^{n}$,

$$
c h^{2} \leq \frac{\mathbf{v}^{T} M \mathbf{v}}{\mathbf{v}^{T} K \mathbf{v}} \leq C,
$$

for constants $c$ and $C$ independent of the step size $h$, and therefore that the eigenvalues of $K^{-1} M$ are contained in an interval of the form $\left[c h^{2}, C\right]$.

Theorem 3 as stated below is proved in [8] using the result (3.2). 
Theorem 3 The eigenvalues of $\hat{S}_{1}^{-1} S$ are bounded as follows:

$$
\lambda\left(\hat{S}_{1}^{-1} S\right) \in\left[\frac{1}{\beta} \tilde{c} h^{4}+1, \frac{1}{\beta} \tilde{C}+1\right],
$$

for constants $\tilde{c}$ and $\tilde{C}$ independent of $h$ and $\beta$.

The Schur complement approximation (3.1) is an improved one, as Theorem 4 demonstrates.

Theorem 4 The eigenvalues of $\hat{S}_{2}^{-1} S$ satisfy the following bound:

$$
\lambda\left(\hat{S}_{2}^{-1} S\right) \in\left[\frac{1}{2}, 1\right]
$$

independently of the values of $h$ and $\beta$.

Proof. First note that $\hat{S}_{2}$ is always non-singular since $M$ is positive definite, and that $K^{-1} M+\sqrt{\beta} I$ is positive real and hence invertible. If we denote the eigenvalues of $\hat{S}_{2}^{-1} S$ by $\mu$, and the corresponding eigenvectors as $\mathbf{x}$, then

$$
\begin{aligned}
\hat{S}_{2}^{-1} S \mathbf{x}=\mu \mathbf{x} & \Rightarrow\left(K M^{-1} K+\frac{1}{\beta} M\right) \mathbf{x}=\mu\left(K+\frac{1}{\sqrt{\beta}} M\right) M^{-1}\left(K+\frac{1}{\sqrt{\beta}} M\right) \mathbf{x} \\
& \Rightarrow\left(K M^{-1} K+\frac{1}{\beta} M\right) \mathbf{x}=\mu\left[K M^{-1} K+\frac{2}{\sqrt{\beta}} K+\frac{1}{\beta} M\right] \mathbf{x} \\
& \Rightarrow\left(I+\frac{1}{\beta} K^{-1} M K^{-1} M\right) \mathbf{x}=\mu\left[I+\frac{2}{\sqrt{\beta}} K^{-1} M+\frac{1}{\beta} K^{-1} M K^{-1} M\right] \mathbf{x} \\
& \Rightarrow\left(\beta I+K^{-1} M K^{-1} M\right) \mathbf{x}=\mu\left[\beta I+2 \sqrt{\beta} K^{-1} M+K^{-1} M K^{-1} M\right] \mathbf{x} \\
& \Rightarrow\left(\beta I+\left(K^{-1} M\right)^{2}\right) \mathbf{x}=\mu\left(K^{-1} M+\sqrt{\beta} I\right)^{2} \mathbf{x} \\
& \Rightarrow\left(K^{-1} M+\sqrt{\beta} I\right)^{-2}\left(\beta I+\left(K^{-1} M\right)^{2}\right) \mathbf{x}=\mu \mathbf{x} .
\end{aligned}
$$

So we deduce for each eigenvalue $\chi$ of $K^{-1} M$, that $\frac{\chi^{2}+\beta}{(\chi+\sqrt{\beta})^{2}}$ is an eigenvalue of $\hat{S}_{2}^{-1} S$. Now since $K^{-1} M$ is similar to a real symmetric matrix $\left(M^{1 / 2} K^{-1} M M^{-1 / 2}\right)$, it is diagonalizable, and hence this describes all eigenvalues of $\hat{S}_{2}^{-1} S$. But $\frac{\chi^{2}+\beta}{(\chi+\sqrt{\beta})^{2}}$ is simply a function of the form $\frac{a^{2}+b^{2}}{(a+b)^{2}}$ with $a$ and $b$ real and positive. It is a simple algebraic task to show that $\frac{1}{2} \leq \frac{a^{2}+b^{2}}{(a+b)^{2}} \leq 1$, and hence that

$$
\lambda\left(\hat{S}_{2}^{-1} S\right) \in\left[\frac{1}{2}, 1\right]
$$

The simplicity of the bound in Theorem 4 is remarkable. Equally remarkable is the fact that, to prove that the bound holds, we did not need to use any spectral properties 
of $K$ or $M$ beside the fact that all eigenvalues of $K^{-1} M$ are real. Hence the bound (3.3) must actually hold for any positive definite self-adjoint operator appropriately approximated by a symmetric matrix, regardless of the order of the elliptic operator.

In Figure 1, we compare the eigenvalue spectra of $\hat{S}_{1}^{-1} S$ and $\hat{S}_{2}^{-1} S$ for the Poisson control problem for a range of values of $\beta$. We can see that for large $\beta$, the approximation $\hat{S}_{1}$ of $S$ is likely to be very effective, as all eigenvalues of $\hat{S}_{1}^{-1} S$ are clustered very close to 1 ; however as $\beta$ becomes smaller the eigenvalues become increasingly spread out, and the convergence of an iterative method will generally suffer as a result. However, for the new approximation $\hat{S}_{2}$, the eigenvalues of $\hat{S}_{2}^{-1} S$ are pinned down into a fixed interval as predicted by (3.3), so an appropriate iterative method should perform well regardless of how small $\beta$ is. Note carefully the vertical scales of the individual plots in Figure 1 to see this.

Note. As the state, control and adjoint are here discretized all using the same piecewise polynomial approximation spaces (as in [12] for example), it would be possible to use the discretized gradient equation to eliminate the second block of the matrix system (1.2), and then solve the remaining $2 \times 2$ block system. The Schur complement of the resulting system would then be exactly the same as that of the original $3 \times 3$ block system, so the approximation $\hat{S}_{2}$ to $S$ detailed in this Section is equally useful in this case.

The approximation to the Schur complement we have given here can be used in effective preconditioners for the three solution approaches we have discussed in Section 2. The use of a fixed number of Chebyshev semi-iteration cycles to approximate mass matrices, along with our approximation $\hat{S}_{2}$ to $S$, can be used as part of a block diagonal, block triangular or symmetric indefinite preconditioner, as in Sections 2.2, 2.3 and 2.4 respectively. Analysis of how well the approximations detailed perform in practice is given in Section 4. In all three cases, the bound (3.3) ensures that the convergence rate of the iteration will be independent of $\beta$; independence with respect to $h$ is ensures if a spectrally equivalent approximation of $K+\frac{1}{\sqrt{\beta}} M$ such as a multigrid process is used.

This new Schur complement approximation thus gives rise to 3 new methods for solving the Poisson control problem. Firstly, we can apply MINRES with the preconditioner (2.3), but now using $\hat{S}_{2}$ to approximate the Schur complement instead of $\hat{S}_{1}$ as in (2.4). Secondly, we may apply Bramble-Pasciak CG preconditioned by (2.5) (using an appropriate choice of $\gamma$, which is typically very close to 1 ), but again replacing the Schur complement approximation $\hat{S}_{1}$ with $\hat{S}_{2}$. Finally, we may apply a non-standard CG method with a symmetric indefinite preconditioner of the form

$$
\hat{\mathcal{P}}_{3}=\left[\begin{array}{ccc}
I & 0 & 0 \\
0 & I & 0 \\
\sigma K \hat{M}^{-1} & -\frac{\sigma}{\beta} M \hat{M}^{-1} & I
\end{array}\right]\left[\begin{array}{ccc}
\frac{1}{\sigma} \hat{M} & 0 & K \\
0 & \frac{\beta}{\sigma} \hat{M} & -M \\
0 & 0 & -\frac{1}{\tau} \hat{S}_{2}
\end{array}\right],
$$

with $\hat{M}$ again an application of Chebyshev semi-iteration to the relevant mass matrix. The bound (3.3) guarantees that we may choose appropriate values $\sigma$ and $\tau$ in the same way as we choose $\gamma$ for the Bramble-Pasciak CG method, using Chebyshev semi-iteration results described in [9]. We test our three new methods in Section 4. 

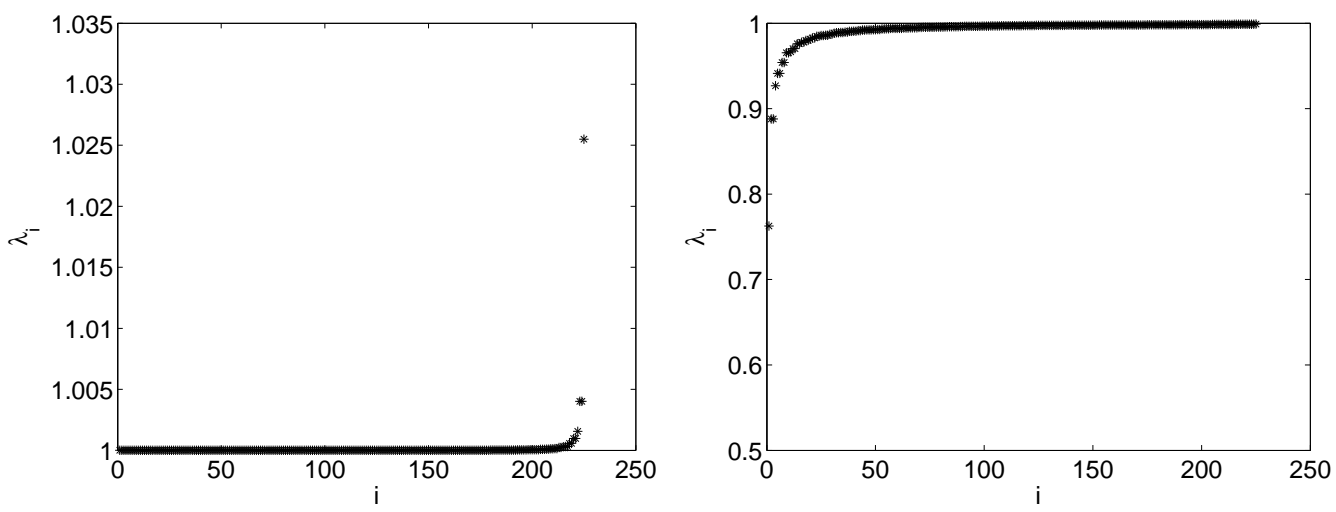

(a) $\lambda_{i}\left(\hat{S}_{1}^{-1} S\right), \beta=10^{-1}, i=1, \ldots, 225$

(b) $\lambda_{i}\left(\hat{S}_{2}^{-1} S\right), \beta=10^{-1}, i=1, \ldots, 225$
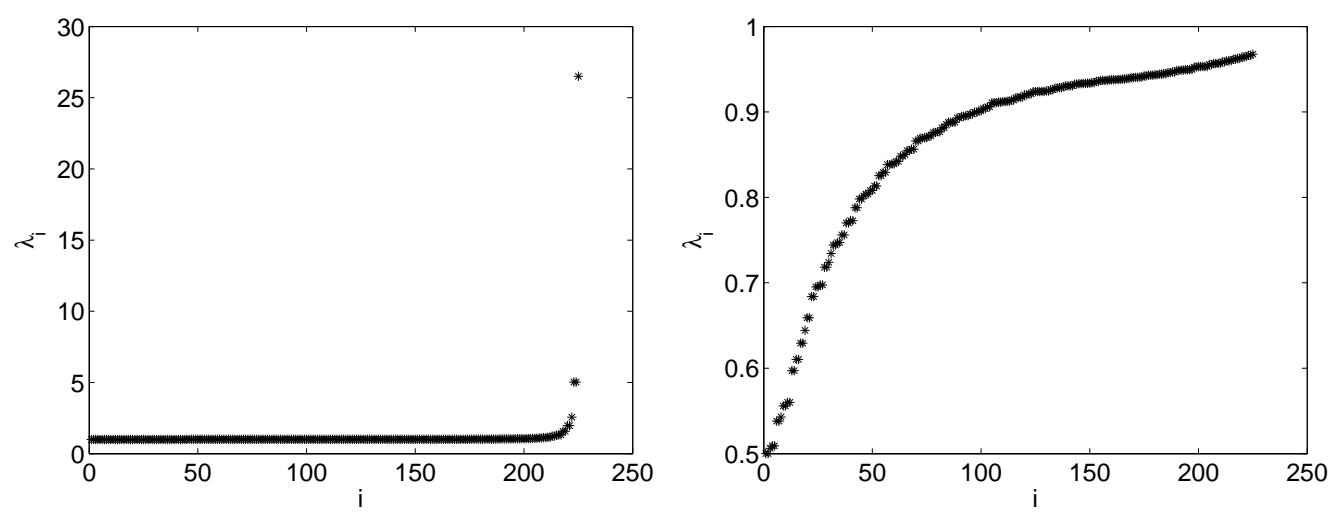

(c) $\lambda_{i}\left(\hat{S}_{1}^{-1} S\right), \beta=10^{-4}, i=1, \ldots, 225$

(d) $\lambda_{i}\left(\hat{S}_{2}^{-1} S\right), \beta=10^{-4}, i=1, \ldots, 225$
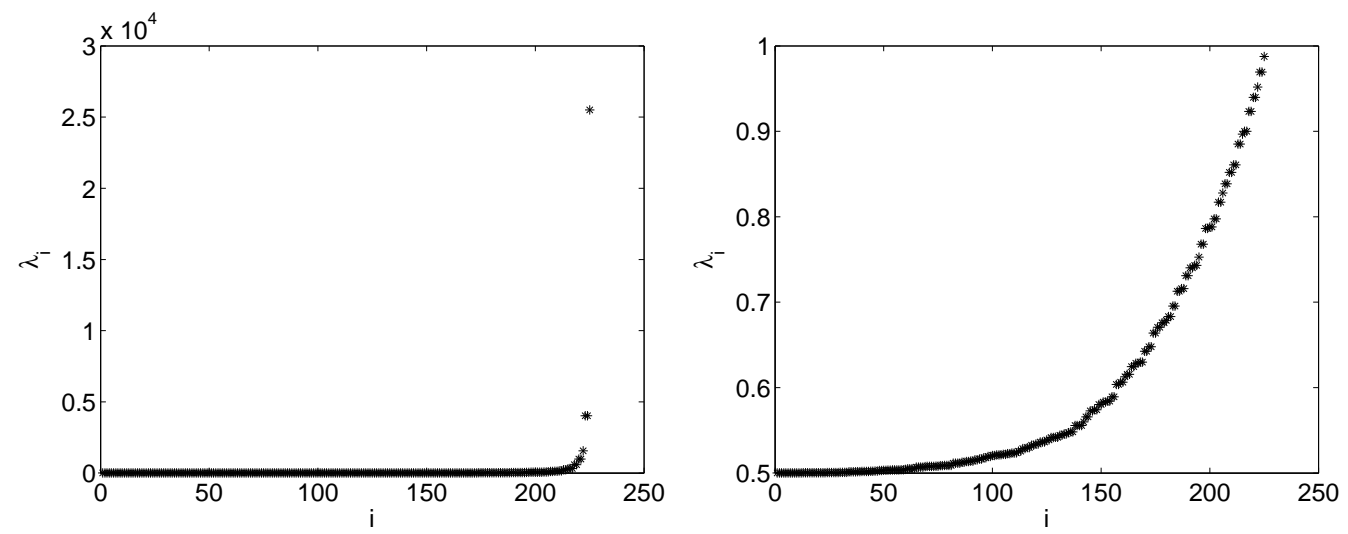

(e) $\lambda_{i}\left(\hat{S}_{1}^{-1} S\right), \beta=10^{-7}, i=1, \ldots, 225$

(f) $\lambda_{i}\left(\hat{S}_{2}^{-1} S\right), \beta=10^{-7}, i=1, \ldots, 225$

Figure 1: Spectra of $\hat{S}_{1}^{-1} S[(\mathrm{a}),(\mathrm{c}),(\mathrm{e})]$ and $\hat{S}_{2}^{-1} S[(\mathrm{~b}),(\mathrm{d}),(\mathrm{f})]$ for $\beta=10^{-1}, \beta=10^{-4}$ and $\beta=10^{-7}$, for an evenly-spaced grid on $\Omega=[0,1]^{2}$ with $h=2^{-4}$ here. 


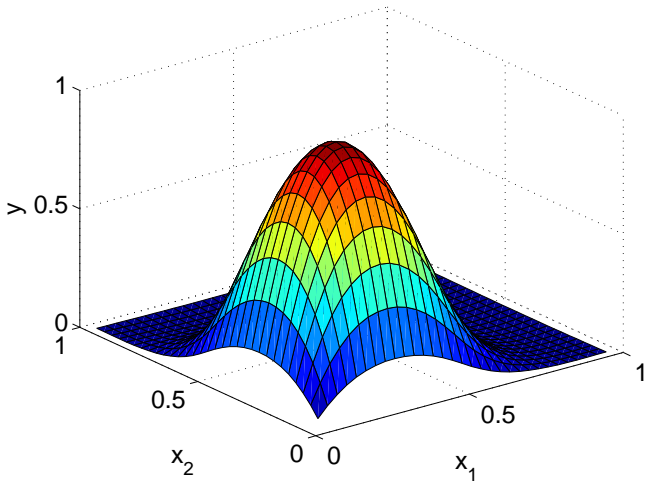

(a) State $y$

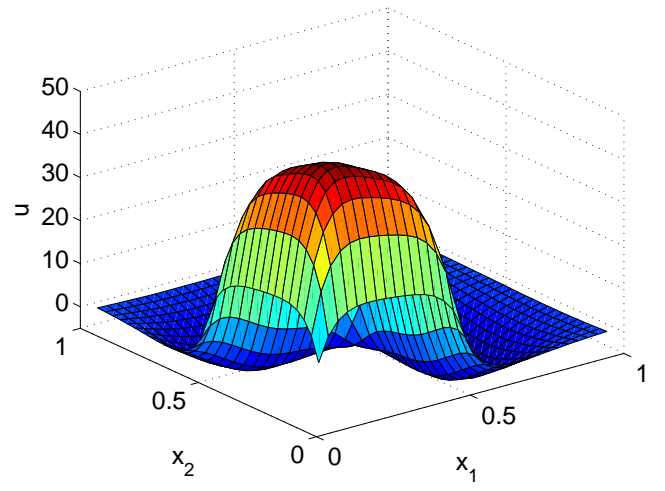

(b) Control $u$

Figure 2: State and control solution to the numerical example in $2 \mathrm{D}$, within $\Omega \backslash \partial \Omega$ with axes $x_{1}$ and $x_{2}$, with $h=2^{-5}$ and $\beta=10^{-4}$.

\section{Numerical Results}

We have demonstrated the theoretical capability of our new Schur complement approximation by the eigenvalue results of Section 3. To illustrate the practical effectiveness of the preconditioners we have proposed, we test them with the block diagonal, block triangular and symmetric indefinite preconditioners proposed in [8], [9] and [10] respectively, and described in Section 2. The problem we consider is given by

$$
\begin{array}{lll}
\min _{y, u} & \frac{1}{2}\|y-\hat{y}\|_{L_{2}(\Omega)}^{2}+\frac{\beta}{2}\|u\|_{L_{2}(\Omega)}^{2} \\
\text { s.t. } & -\nabla^{2} y=u & \text { in } \Omega, \\
y=0 & \text { on } \partial \Omega,
\end{array}
$$

where $\Omega=[0,1]^{2}, \partial \Omega$ denotes its boundary, and $\hat{y}$ is given by

$$
\hat{y}= \begin{cases}1 & \text { in }\left[0, \frac{1}{2}\right]^{2}=: \Omega_{1}, \\ 0 & \text { in } \Omega \backslash \Omega_{1}\end{cases}
$$

The solution for the state and control of this problem when $\beta=10^{-4}$ is shown in Figure 2 .

In Table 1, we compare the number of MINRES iterations required to solve this problem to a tolerance of $10^{-6}$ using block diagonal preconditioner (2.3) for a range of $h$ and $\beta$, using the approximations $\hat{S}_{1}$ and $\hat{S}_{2}$ to the Schur complement.

To exemplify that our method is equally applicable in three dimensions, we present results for solving the problem above, except with $\Omega=[0,1]^{3}$ and $\Omega_{1}=\left[0, \frac{1}{2}\right]^{3}$, using MINRES with block diagonal preconditioner (2.3), in Table 2. In Table 3, we show the number of Bramble-Pasciak Conjugate Gradient iterations required to solve the test problem using the block triangular preconditioner (2.5), approximating $S$ by $\hat{S}_{1}$ and $\hat{S}_{2}$. Finally in Table 4, we compare the number of Conjugate Gradient iterations with $\hat{A}$ and 


\begin{tabular}{|c|c|c|c|c|c||c|c|c|c|}
\hline \multicolumn{2}{|c||}{} & \multicolumn{4}{c||}{ Approximation $\hat{\mathcal{S}}_{1}$} & \multicolumn{4}{c|}{ Approximation $\hat{\mathcal{S}}_{2}$} \\
\cline { 3 - 9 } \multicolumn{2}{|c|}{} & \multicolumn{3}{|c||}{$\beta$} & \multicolumn{4}{c|}{$\beta$} \\
\cline { 2 - 9 } \multicolumn{2}{|c|}{} & $10^{-2}$ & $10^{-4}$ & $10^{-6}$ & $10^{-8}$ & $10^{-2}$ & $10^{-4}$ & $10^{-6}$ & $10^{-8}$ \\
\hline \hline \multirow{5}{*}{$h$} & $2^{-4}$ & 9 & 21 & 73 & 248 & 13 & 16 & 15 & $-\dagger$ \\
& $2^{-5}$ & 9 & 20 & 82 & 484 & 13 & 17 & 16 & 15 \\
& $2^{-6}$ & 9 & 22 & 85 & 592 & 13 & 17 & 16 & 16 \\
& $2^{-7}$ & 11 & 22 & 84 & 619 & 13 & 17 & 16 & 16 \\
& $2^{-8}$ & 11 & 21 & 85 & 646 & 15 & 17 & 17 & 16 \\
\hline
\end{tabular}

Table 1: Number of MINRES iterations with block diagonal preconditioner (2.3) required to solve the test problem in 2D, using Q1 basis functions for state and control, for a variety of $h$ and $\beta$. Results are given when the Schur complement is approximated as $\hat{S}_{1}$ and $\hat{S}_{2}$.

\begin{tabular}{|c|c||c|c|c|c||c|c|c|c|}
\hline \multicolumn{2}{|c||}{} & \multicolumn{4}{c||}{ Approximation $\hat{\mathcal{S}}_{1}$} & \multicolumn{4}{c|}{ Approximation $\hat{\mathcal{S}}_{2}$} \\
\cline { 2 - 9 } \multicolumn{2}{|c|}{} & \multicolumn{3}{c||}{$\beta$} & \multicolumn{4}{c|}{$\beta$} \\
\cline { 2 - 9 } \multicolumn{2}{|c|}{} & $10^{-1}$ & $10^{-3}$ & $10^{-5}$ & $10^{-7}$ & $10^{-1}$ & $10^{-3}$ & $10^{-5}$ & $10^{-7}$ \\
\hline \hline \multirow{4}{*}{$h$} & $2^{-2}$ & 8 & 12 & 26 & 28 & 10 & 14 & $-\dagger$ & $-\dagger$ \\
& $2^{-3}$ & 8 & 12 & 42 & 130 & 10 & 16 & 14 & $-\dagger$ \\
& $2^{-4}$ & 8 & 12 & 48 & 272 & 12 & 17 & 15 & 13 \\
& $2^{-5}$ & 10 & 14 & 49 & 341 & 12 & 18 & 16 & 16 \\
\hline
\end{tabular}

Table 2: Number of MINRES iterations with block diagonal preconditioner (2.3) required to solve the test problem in 3D, using Q1 basis functions for state and control, for a variety of $h$ and $\beta$. Results are given when the Schur complement is approximated as $\hat{S}_{1}$ and $\hat{S}_{2}$.

$\hat{S}$ defined by (2.6), (2.7) as described in [10], with the number of iterations required when using the preconditioner (3.4) with Schur complement approximation $\hat{S}_{2}$ (except that we use Chebyshev semi-iterations to approximate a mass matrix rather than Gauss-Seidel iteration as in [10]; this equally improves both approaches).

On each occasion, we have used 20 Chebyshev semi-iterations to approximate a mass matrix, and 2 algebraic multigrid V-cycles with 2 pre- and post- Jacobi smoothing steps whenever a multigrid solve is required. We employ the Harwell Science Library code HSL_MI20 [3] via a MATLAB interface for the (algebraic) multigrid cycles. In Tables 1-4, $\dagger$ denotes that the coarsening failed when the code HSL_MI20 was applied to $K+\frac{1}{\sqrt{\beta}} M$, which occurs only when $h$ is large and $\beta$ is very small. This clearly occurs in relatively few cases, and is caused by the presence of positive off-diagonal entries. The use of large $h$ and small $\beta$ is in any case not an interesting practical parameter regime.

Tables 1-4 clearly illustrate the potency of our new Schur complement approximation; $h$ and $\beta$-independent convergence is clearly exhibited in all cases. In Tables $1-3$, we can see that for larger values of $\beta$, the approximation of the Schur complement $\hat{S}_{1}$ is 


\begin{tabular}{|c|c|c|c|c|c|c|c|c|c|}
\hline & \multicolumn{4}{|c|}{$\frac{\text { Approximation } \hat{\mathcal{S}}_{1}}{\beta}$} & \multicolumn{4}{|c|}{$\frac{\text { Approximation } \hat{\mathcal{S}}_{2}}{\beta}$} \\
\hline & & $10^{-2}$ & $10^{-4}$ & $10^{-6}$ & $10^{-8}$ & $10^{-2}$ & $10^{-4}$ & $10^{-6}$ & $10^{-8}$ \\
\hline \multirow{5}{*}{$h$} & $2^{2-4}$ & 6 & 18 & $\overline{100}$ & 600 & 9 & 12 & $\overline{14}$ & -1 \\
\hline & $2^{-5}$ & 7 & 19 & 106 & 815 & 10 & 13 & 15 & 16 \\
\hline & $2^{-6}$ & 7 & 19 & 109 & $>1500$ & 10 & 14 & 15 & 16 \\
\hline & $2^{-7}$ & 7 & 19 & 117 & 1334 & 11 & 14 & 16 & 17 \\
\hline & $2^{-8}$ & 8 & 20 & 116 & 1269 & 11 & 14 & 16 & 17 \\
\hline
\end{tabular}

Table 3: Number of Bramble-Pasciak CG iterations with block triangular preconditioner (2.5) required to solve the test problem in 2D, using Q1 basis functions for state and control, for a variety of $h$ and $\beta$. Results are given when the Schur complement is approximated using $\hat{S}_{1}$ and $\hat{S}_{2}$.

\begin{tabular}{|c|c|c|c|c|c|c|c|c|c|}
\hline & \multirow{2}{*}{\multicolumn{4}{|c|}{$\frac{\text { Approximation as in }[10]}{\beta}$}} & \multirow{2}{*}{\multicolumn{4}{|c|}{$\frac{\text { Approximation } \hat{\mathcal{S}}_{2}}{\beta}$}} \\
\hline & & & & & & & & & \\
\hline & & $10^{-2}$ & $10^{-4}$ & $10^{-6}$ & $10^{-8}$ & $10^{-2}$ & $10^{-4}$ & $10^{-6}$ & $10^{-8}$ \\
\hline \multirow{5}{*}{$h$} & $2^{2-4}$ & $\overline{11}$ & 14 & 13 & $-{ }^{2} \dagger$ & 8 & 11 & 12 & -1 \\
\hline & $2^{-5}$ & 11 & 14 & 16 & 14 & 9 & 13 & 12 & 14 \\
\hline & $2^{-6}$ & 11 & 15 & 17 & 16 & 9 & 13 & 13 & 15 \\
\hline & $2^{-7}$ & 12 & 16 & 18 & 19 & 9 & 14 & 13 & 15 \\
\hline & $2^{-8}$ & 12 & 16 & 18 & 20 & 10 & 14 & 14 & 15 \\
\hline
\end{tabular}

Table 4: Number of CG iterations with symmetric indefinite preconditioner (2.8) required to solve the test problem in 2D, using Q1 basis functions for state and control, for a variety of $h$ and $\beta$. Results are given with $\hat{A}$ and $\hat{S}$ as stated in (2.6), (2.7) and detailed in [10], and using the preconditioner (3.4) with $\hat{S}$ approximated using $\hat{S}_{2}$.

marginally more effective than the approximation $\hat{S}_{2}$, as the eigenvalues of $\hat{S}_{1}^{-1} S$ are more clustered than those of $\hat{S}_{2}^{-1} S$ (see Figure 1). However, as $\beta$ gets smaller, it is easily observable that as the eigenvalues of $\hat{S}_{1}^{-1} S$ become less clustered but those of $\hat{S}_{2}^{-1} S$ remain in the interval $\left[\frac{1}{2}, 1\right]$, our new Schur complement approximation performs far better. Table 4 also illustrates that our approximations of mass matrices and Schur complement leaves us with a preconditioner that is competitive with the symmetric indefinite preconditioner proposed in [10].

\section{Conclusions}

In this paper, we have first reviewed three solvers previously proposed for the Poisson control problem: a MINRES approach with a block diagonal preconditioner, a BramblePasciak CG solver with a block triangular preconditioner, and a CG method with a 
symmetric indefinite preconditioner. We have also detailed the relevant approximations to the mass matrices and Schur complements which were used in these solvers. We then proposed a new approximation to the Schur complement, and proved that $\beta$-independence as well as mesh-independence should be achieved when it is applied.

The new Schur complement approximation, combined with a Chebyshev semi-iteration method to approximate the relevant mass matrices, is readily built into the symmetric indefinite preconditioner discussed in [10], and in the block diagonal and block triangular preconditioned solvers discussed in [8] and [9]. In numerical experiments, our suggested preconditioners are demonstrated to give rapid parameter-independent convergence in each case.

Acknowledgements. The first author would like to thank the Engineering and Physical Sciences Research Council (EPSRC) for financial support.

\section{References}

[1] Benzi M, Golub GH, Liesen J. Numerical solution of saddle point problems. Acta Numerica 2005; 14:1-137.

[2] Borzi A, Schulz V. Multigrid methods for PDE optimization. SIAM Review 2009; 51(2):361-395.

[3] Boyle J, Mihajlovic MD, Scott JA. HSL_MI20: An efficient AMG preconditioner for finite element problems in 3D. International Journal for Numerical Methods in Engineering 2010; 82:64-98.

[4] Bramble JH, Pasciak JE. A preconditioning technique for indefinite systems resulting from mixed approximations of elliptic problems. Mathematics of Computation 1988; 50(181):1-17.

[5] Elman HC, Silvester DJ, Wathen AJ. Finite elements and fast iterative solvers: with applications in incompressible fluid dynamics. Numerical Mathematics and Scientific Computation Series. Oxford University Press: New York, 2005.

[6] Murphy MF, Golub GH, Wathen AJ. A note on preconditioning for indefinite linear systems. SIAM Journal on Scientific Computing 2000; 21(6):1969-1972.

[7] Paige CC, Saunders MA. Solutions of sparse indefinite systems of linear equations. SIAM Journal on Numerical Analysis 1975; 12(4):617-629.

[8] Rees T, Dollar HS, Wathen AJ. Optimal solvers for PDE-constrained optimization. SIAM Journal on Scientific Computing 2010; 32(1):271-298.

[9] Rees T, Stoll M. Block triangular preconditioners for PDE-constrained optimization. To appear in Numerical Linear Algebra with Applications; DOI: 10.1002/nla.693, 2010 . 
[10] Schöberl J, Zulehner W. Symmetric indefinite preconditioners for saddle point problems with applications to PDE-constrained optimization problems. SIAM Journal on Matrix Analysis and Applications 2007; 29(3):752-773.

[11] Stoll M, Wathen A. Combination preconditioning and the Bramble-Pasciak ${ }^{+}$preconditioner. SIAM Journal on Matrix Analysis and Applications 2008; 30(2):582608 .

[12] Takacs S, Zulehner W. Computing smoothing rates of collective point smoothers for optimal control problems using symbolic computation. Technical Report No. 201009, Doctoral Program in Computational Mathematics, Johannes Kepler University Linz.

[13] Thorne HS. Properties of linear systems in PDE-constrained optimization. Part I: Distributed control. Rutherford Appleton Laboratory Technical Report RAL-TR2009-017, submitted to SIAM Journal on Matrix Analysis and Applications.

[14] Thorne HS. Properties of linear systems in PDE-constrained optimization. Part II: Neumann boundary control. Rutherford Appleton Laboratory Technical Report RALTR-2009-018, submitted to SIAM Journal on Matrix Analysis and Applications.

[15] Tröltzsch F. Optimal control of partial differential equations: Theory, methods and applications. Graduate Series in Mathematics. American Mathematical Society, 2010 .

[16] Wathen AJ. Realistic eigenvalue bounds for the Galerkin Mass Matrix. IMA Journal of Numerical Analysis 1987; 7(4):449-457.

[17] Wathen AJ, Rees T. Chebyshev semi-iteration in preconditioning for problems including the mass matrix. Electronic Transactions on Numerical Analysis 2009; 34:125-135.

[18] Zulehner W. Analysis of iterative methods for saddle point problems: a unified approach. Mathematics of Computation 2002; 71:479-505. 